\title{
Adduct Formation in Quantitative Bioanalysis: Effect of Ionization Conditions on Paclitaxel
}

\author{
Kjell A. Mortier, Guo-Fang Zhang, Carlos H. Van Peteghem, \\ and Willy E. Lambert \\ Laboratory of Toxicology, Ghent University, Ghent, Belgium
}

\begin{abstract}
Quantitative analysis of target compounds with liquid chromatography atmospheric pressure ionization mass spectrometry is sometimes hampered by adduct formation. In these situations, cationization with alkali metal ions instead of proton addition is often observed in the positive ion mode. This work studies the process of adduct formation and investigates potential strategies to control this phenomenon. Paclitaxel, a pharmaceutical chemotherapeutic agent, was used as a model compound. Electrospray (ESI), atmospheric pressure chemical ionization (APCI) and sonic spray ionization (SSI) are evaluated and compared. The work was performed on two different instruments, allowing the evaluation of different ionization behavior for different source design for electrospray, if any. Different mobile phase additives were compared, including acetic acid, formic acid, ammonium formate, and a range of primary amines. Continuous infusion was used for a fast screening, to detect optimal conditions. These were then further investigated in detail by LC-MS. The results indicate that electrospray is the more sensitive interface for this compound on the investigated apparatus. Unacceptable quantitative data were acquired without additives in the mobile phase. Generally, additives increased the reproducibility significantly. A response of mainly one ion was achieved with dodecylamine/acetic acid and acetic acid/sodium acetate. The data also point out the importance of evaluating adduct formation for compounds prone to this phenomenon during method development, especially in view of accurate quantitation. (J Am Soc Mass Spectrom 2004, 15, 585-592) @ 2004 American Society for Mass Spectrometry
\end{abstract}

$\mathrm{D}$ ue to recent advances in atmospheric pressure ionization (API) techniques, liquid chromatography mass spectrometry (LC-MS) has opened a new chapter in today's analytical chemistry. Many companies and institutes are successfully using LC-MS for high throughput analysis of lowly concentrated target compounds. Nevertheless, some challenges still remain when performing quantitative LC-MS. An important issue is matrix suppression, a less efficient ionization of the target compound in the presence of other molecules. This phenomenon is well recognized and described in an increasing number of publications [1-4]. Another complexity in quantitative LC-MS is adduct formation. Generally, electrospray (ESI) or atmospheric pressure chemical ionization (APCI) result in protonated $[\mathrm{M}+\mathrm{H}]^{+}$molecules in the positive ion mode or deprotonated $[\mathrm{M}-\mathrm{H}]^{-}$molecules in the negative ion mode. However, for some molecules undergoing ionization in the positive ion mode, adduct ions like $[\mathrm{M}+\mathrm{Na}]^{+},[\mathrm{M}+\mathrm{K}]^{+}$or $\left[\mathrm{M}+\mathrm{NH}_{4}\right]^{+}$are abundant in the spectra [5-11]. The exact mechanism of

Published online February 5, 2004

Address reprint requests to Dr. W. E. Lambert, Laboratory of Toxicology, Ghent University, Harelbekestraat 72, B-9000 Ghent, Belgium. E-mail: Willy.Lambert@UGent.be adduct formation still has to be elucidated. In some cases at least, carboxyl or carbonyl ether or ester groups in a molecule are believed to be responsible for the binding to alkali metal ions. Difficulties arise when developing quantitative LC-API-MS procedures. "What adduct ion to use for the traditional multiple reaction monitoring (MRM)?" and "Is the adduct process reproducible?" are key questions when a quantitative assay is required. Multiple approaches have been described in literature. A first theoretical approach is to ignore the different adduct forms and measure the adduct with the highest response. When measuring only the $[\mathrm{M}+$ $\left.\mathrm{NH}_{4}\right]^{+}$in the presence of other adducts, $\mathrm{Li}$ and coworkers obtained a relative standard deviation (RSD) of $17 \%$ for a quantitative assay of ginkgolides and bilobalide [5]. Summation of all adduct ions significantly improved the situation to an acceptable RSD of $6 \%$. This shows that adduct formation can give rise to large variations and unreliable results when the process is not controlled. Summation, however, complicates MS/MS experiments, thus compromising selectivity. Although performing MRM on the different adducts simultaneously can be considered, it is impractical to add the responses of the different MS/MS traces. In addition, summation assumes that the response factor for all adduct ions is equal, which has not been demonstrated 
to our knowledge. A convenient solution to this problem would be to use an isotopic labeled internal standard, as this compound would most probably produce a similar adduct distribution, and at least partly compensate for variations in adduct formation. Unfortunately, the availability of such compounds is sometimes problematic. Obtaining a pronounced and reproducible formation of a single ion is of course the ideal situation regarding to sensitivity as well as reproducibility. Successful attempts have been made to replace all adducts by one desired adduct ion with primary amines [6-8]. Another approach involved the addition of alkali metal complexation products, such as crown ethers [9]. In oligonucleotide analysis, the addition of imidazole and triethylamine or piperidine has proven to be successful to reduce the number of adducted cations. Another well documented approach in this research area is the ethanol precipitation in the presence of ammonium acetate [10]. One could also try to exclude sodium from the ionization process by using ultra pure, thoroughly deionized water. This seems quite laborious due to the ubiquitous presence of sodium, often originating from the glassware, stainless steel and/or as impurity in chemicals or solvents. The opposite approach, the use of sodium adducts for quantification by the addition of sodium acetate to the mobile phase, has also been described [11].

The goal of this study is to evaluate various ionization parameters and sources to obtain a reproducible ionization when adduct ion formation is observed. Especially the use of different ionization techniques and mobile phase additives were considered. Paclitaxel (PAC), a widespread drug used for the treatment of several types of cancer, was selected for this purpose. Paclitaxel has a high molecular weight and is a very hydrophobic molecule (Structure 1). It is a natural compound present in the bark of Taxus brevifolia. To the best of our knowledge, this is the first paper investigating adduct ion formation of paclitaxel. In literature, quantitative analysis of paclitaxel by the analysis of $[\mathrm{PAC}+\mathrm{H}]^{+}$has been reported with acetic acid as mobile phase additive [12]. Nevertheless we observed strong adduct formation of paclitaxel with sodium and potassium under such conditions. Possibly, different instrumentation design accounts at least partly for some differences. Although adduct formation is compound and instrument dependent, we believe that our experimental approach and results can be useful for method development for other substances susceptible to adduct formation.

\section{Experimental}

\section{Instrumentation}

The following instrumentation was used: a LaChrom separation module (Merck, Darmstadt, Germany) including a L-7100 Low-Pressure Gradient Pump, L-7200 Autosampler and a D-7000 interface module. The mod-

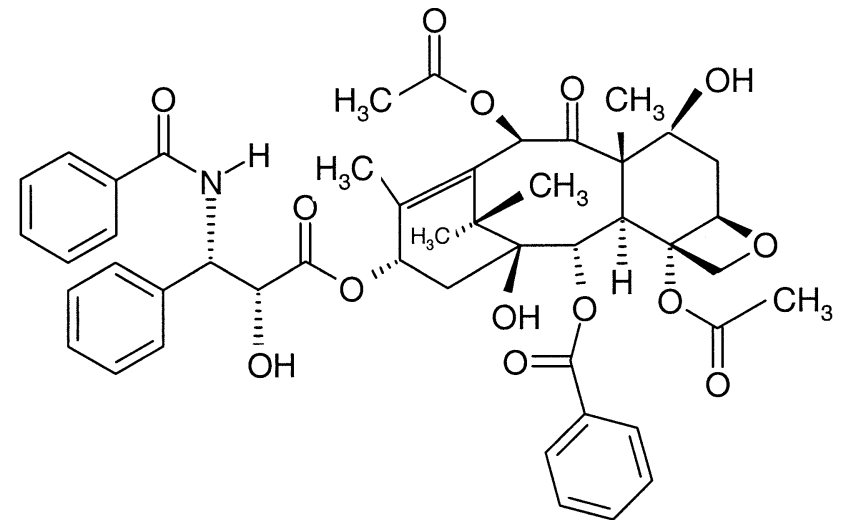

Structure 1. Paclitaxel (molecular weight: 853)

ule was controlled by LC/3DQ-MS System Manager Software under Windows NT 4.0 and connected to a M-8000 ion-trap based mass spectrometer equipped with an ESI, APCI and SSI (sonic spray ionization) source (Merck-Hitachi, Darmstadt, Germany). General conditions on M-8000: focus: $30 \mathrm{~V}$, drift: $30 \mathrm{~V}$, multiplier voltage: $400 \mathrm{~V}$. Nitrogen was used as nebulizing gas and high purity helium was used as buffer gas to trap ions in the mass analyzer at $300 \mathrm{kPa}$. Scan range from $\mathrm{m} / \mathrm{z}$ $90-1100$, the accumulation time was automatically adjusted by a variation in the ion abundance with "Automatic Sensitivity Control" (ASC) with a maximum of $500 \mathrm{~ms}$. Specific operating conditions for SSI; plate temperature: $250{ }^{\circ} \mathrm{C}$, aperture 1 temperature: $140{ }^{\circ} \mathrm{C}$, aperture 2 temperature: $120^{\circ} \mathrm{C}$ and ESI; aux gas heater: $400{ }^{\circ} \mathrm{C}$, aperture 1 temperature: $150{ }^{\circ} \mathrm{C}$, aperture 2 temperature: $120{ }^{\circ} \mathrm{C}$, needle: $3 \mathrm{kV}$.

A second system consisted of a Waters Alliance 2790 Separation Module (Milford, MA) controlled by Masslynx software from Micromass (Manchester, UK). Mass spectrometric detection on this system was performed on a quadrupole time of flight (QTOF) mass spectrometer with an ESI and APCI Z-spray source (Micromass, Manchester, UK). ESI conditions on QTOF: needle: $3 \mathrm{kV}$, cone: 25 or $40 \mathrm{~V}$, source block temperature: $120^{\circ} \mathrm{C}$, desolvation temperature: $320^{\circ} \mathrm{C}$ and APCI conditions: corona: $2500 \mathrm{~V}$, cone: 25 or $40 \mathrm{~V}$, source temperature: $120^{\circ} \mathrm{C}$, APCI probe temperature: $500^{\circ} \mathrm{C}$.

A syringe pump (Harvard, Quebec, Canada) with a $500-\mu \mathrm{L}$ syringe, (Hamilton, Bonaduz, Switzerland) was used for direct infusion at $200 \mu \mathrm{L} / \mathrm{min}$. HPLC column: Synergy MAX-RP $4 \mu \mathrm{m}, 80 \mathrm{~A}, 150 \times 2.00 \mathrm{~mm}$ (Phenomenex, CA).

\section{Reagents and Standards}

All solvents and reagents were obtained from VWR (Leuven, Belgium) or Sigma (Bornem, Belgium). Stock solutions of paclitaxel (Sigma-Aldrich, Bornem, Belgium) were prepared by accurately weighing $10 \mathrm{mg}$ powder and dissolving in $10 \mathrm{ml}$ of methanol. 
Table 1. Absolute response of paclitaxel adduct ions (in Arbitrary Units, M-8000 and QTOF units are not comparable) after infusion with common additives

\begin{tabular}{|c|c|c|c|c|c|}
\hline \multicolumn{6}{|c|}{ M-8000 } \\
\hline$E S I$ & $\mathrm{MeOH}$ & $A A$ & $F A$ & $A m F A, p H 5$ & $A m F A, p H 7$ \\
\hline $\mathrm{PAC}+\mathrm{Na}$ & 130000 & 115000 & 90000 & 35000 & 50000 \\
\hline $\mathrm{PAC}+\mathrm{K}$ & 35000 & 65000 & 55000 & 28000 & 25000 \\
\hline$S S I$ & $\mathrm{MeOH}$ & $A A$ & $F A$ & $A m F A, p H 5$ & $A m$ FA, $p H 7$ \\
\hline $\mathrm{PAC}+\mathrm{Na}$ & 86600 & 51000 & 27600 & 10500 & 12000 \\
\hline $\mathrm{PAC}+\mathrm{K}$ & 103000 & 85300 & 40000 & 8800 & 15100 \\
\hline \multicolumn{6}{|c|}{ QTOF } \\
\hline$E S I$ & $\mathrm{MeOH}$ & $A A$ & $F A$ & $A m F A, p H 5$ & $A m F A, p H 7$ \\
\hline $\mathrm{PAC}+\mathrm{H}$ & 20 & 30 & 30 & 260 & 240 \\
\hline $\mathrm{PAC}+\mathrm{Na}$ & 3400 & 3900 & 3200 & 1200 & 2300 \\
\hline $\mathrm{PAC}+\mathrm{K}$ & 2500 & 1250 & 900 & 450 & 900 \\
\hline$A P C l$ & $\mathrm{MeOH}$ & $A A$ & $F A$ & $A m F A, p H 5$ & $A m F A, p H$ \\
\hline $\mathrm{PAC}+\mathrm{H}$ & 10 & 10 & 10 & 15 & 15 \\
\hline $\mathrm{PAC}+\mathrm{Na}$ & 2600 & 1000 & 800 & 1000 & 800 \\
\hline$P A C+K$ & 800 & 750 & 580 & 400 & 400 \\
\hline
\end{tabular}

MeOH: MeOH/Water 70/30 by vol., AA: acetic acid, FA: formic acid, Am FA: ammonium formate, all at 10 mM. Cone voltage QTOF: 40 V.

\section{Methods}

Two experimental setups were developed. Initially, for a screening of additives, a mixture of paclitaxel and the respective additives was infused with a syringe into the MS. The paclitaxel concentration amounted $0.5 \mu \mathrm{g} / \mathrm{mL}$ and the flow was set at $0.2 \mathrm{~mL} / \mathrm{min}$. Using this solution, the interfaces were tuned to optimum sensitivity for the paclitaxel sodium adduct $[\mathrm{PAC}+\mathrm{Na}]^{+}$. Acetic acid, formic acid and ammonium formate at $\mathrm{pH} 5$ and 7 were evaluated at $10 \mathrm{mM}$, primary amines were introduced at $1 \mathrm{mM}$ with acetic acid $(1 \mathrm{mM})$ to lower the $\mathrm{pH}$. Results are calculated by the signal height at different points $(n=3)$.

After infusion, evaluation of adduct formation was performed with a chromatographic method with methanol/water (70/30 by vol.) and the respective additives as mobile phase. Three samples, differing in alkali metal content and type, but with an equal concentration of paclitaxel $(1 \mu \mathrm{g} / \mathrm{mL})$ were cross injected $(n=8)$. $\mathrm{NaCl}$ $(17 \mathrm{mM})$ was added to the first sample $(\mathrm{S}+\mathrm{NaCl}), \mathrm{KCl}$ $(4 \mathrm{mM})$ to the second $(\mathrm{S}+\mathrm{KCl})$, and nothing to the third (S), this to mimic real samples with varying concentrations of alkali metals. This allowed to observe the effect of alkali metals on adduct formation. The results are expressed as absolute response (response) i.e., the response of the adduct ion in arbitrary units (or peak area for chromatography) or relative response (per cent response) i.e., the response of one adduct ion divided by the sum of responses of all adduct ion forms times 100 .

\section{Results and Discussion}

\section{Infusion Experiments with Traditional Additives}

Continuous infusion was performed on the M-8000 ion trap based LC-MS (Merck-Hitachi) equipped with ESI, SSI and APCI and on the quadrupole Time-of-Flight (QTOF) from Micromass equipped with ESI and APCI interface. The APCI interface on the M-8000 did not generate a significant response for paclitaxel under various conditions, so this interface was excluded for further experiments. The optimized infusion conditions (see the Experimental section) of the remaining four interfaces (ESI, SSI on M-8000; ESI, APCI on QTOF) were applied to observe the response with some common LC-MS additives (see experimental), of which the results are depicted in Table 1.

On the M-8000, both ESI and SSI resulted in spectra dominated by the $[\mathrm{PAC}+\mathrm{Na}]^{+}$and $\left[\mathrm{PAC}+\mathrm{K}^{+}\right.$ion. $\mathrm{No}[\mathrm{PAC}+\mathrm{H}]^{+}$was observed with any of the additives. Changing from $\mathrm{pH} 5$ to $\mathrm{pH} 7$ did not have a remarkable influence on the ionization. All additives (except acetic acid) caused a lower signal intensity, probably due to ion suppression by the additive.

On the QTOF, the spectra are again dominated by sodium and potassium adduct ions. For the APCI source, this is somewhat surprising, as it is often observed that ionization with this interface results in less adduct formation [13]. Electrospray ionization on the QTOF resulted in substantial quantities of [PAC + $\mathrm{H}]^{+}$in the presence of ammonium formate. The addi- 
Table 2. Absolute (in Arbitrary Units, M-8000 and QTOF units are not comparable) and relative response (response of primary amine adduct divided by total response of all paclitaxel adduct ions times 100, between brackets) of the paclitaxel primary amine adduct after paclitaxel infusion with primary amines $(1 \mathrm{mM})$ and acetic acid $(1 \mathrm{mM})$

\begin{tabular}{lcccrr}
\hline M-8000 & MA1/AA1 & PA1/AA1 & HX1/AA1 & NA1/AA1 & DDA1/AA1 \\
\hline \hline ESI & $62183(40 \%)$ & $107167(51 \%)$ & $95800(55 \%)$ & $147767(73 \%)$ & $130600(76 \%)$ \\
SSI & $12267(41 \%)$ & $20000(28 \%)$ & $39167(34 \%)$ & $27278(71 \%)$ & $31933(74 \%)$ \\
\hline QTOF & MA1/AA1 & PA1/AA1 & HX1/AA1 & NA1/AA1 & DDA1/AA1 \\
\hline ESI & $476(35 \%)$ & $1437(50 \%)$ & $2013(71 \%)$ & $2210(77 \%)$ & $1867(69 \%)$ \\
APCI & $70(8 \%)$ & $130(16 \%)$ & $200(23 \%)$ & $210(24 \%)$ & $250(29 \%)$ \\
\hline
\end{tabular}

Cone voltage QTOF: 40 V. MA: methylamine, PA: propylamine, HX: hexylamine, NA: nonylamine, DDA: dodecylamine, AA: acetic acid.

tion of formic or acetic acid on the other hand, did not show such a noticeable formation of $[\mathrm{PAC}+\mathrm{H}]^{+}$. Considering the large $\mathrm{pH}$ difference between these acids and ammonium formate $\mathrm{pH} 7$, it is evident that a low $\mathrm{pH}$ is not the critical factor for the production of $[\mathrm{PAC}+\mathrm{H}]^{+}$. The mechanism through which ammonium acetate results in $[\mathrm{PAC}+\mathrm{H}]^{+}$might be the formation of $\left[\mathrm{PAC}+\mathrm{NH}_{4}\right]^{+}$ions and subsequent dissociation into $[\mathrm{PAC}+\mathrm{H}]^{+}$and $\mathrm{NH}_{3}$. Similar observations have also been described for digitoxin by Zhou and Hamburger [14].

The cone voltage has a remarkable influence on the response observed for $\left[\mathrm{PAC}+\mathrm{H}^{+}\right.$and $[\mathrm{PAC}+\mathrm{Na}]^{+}$ on the QTOF. A cone voltage of $25 \mathrm{~V}$ gives a maximal response for $\left[\mathrm{PAC}+\mathrm{H}^{+}\right.$while a cone voltage of $40 \mathrm{~V}$ results in a maximal signal for $[\mathrm{PAC}+\mathrm{Na}]^{+}$. A great loss of signal is observed for [PAC $+\mathrm{H}^{+}$at this higher cone voltage, due to up front fragmentation. This implies that different adduct ions of a given molecule should be viewed as ions with separate optimum ionization settings. Different behavior of adduct ions of a given molecule are also observed with sources from other manufacturers [11].

On all sources, acetic acid provided a more efficient ionization as compared to formic acid. Finally, none of the additives provided a pronounced formation of one ion, on any of the interfaces. Based on these infusion experiments, the tested common LC-MS additives were not suitable for a pronounced ionization to one single ion.

\section{Infusion Experiments with Primary Amines}

Another option explored with infusion consisted of the addition of primary amines (PrAm) to the liquid phase. A range of primary amines was selected, some based on literature reports and some to obtain a distribution of primary amines with varying chain lengths. The following compounds were evaluated: methylamine, propylamine, hexylamine, nonylamine, dodecylamine and octadecylamine. These are all quite strong basic compounds, with pKas of approximately 10.7 (calculated using ACD software Solaris V4.67). As such compounds could cause column deterioration and paclitaxel breakdown, an acid had to be added to lower the $\mathrm{pH}$. Initial experiments established $1 \mathrm{mM}$ of the primary amine and $1 \mathrm{mM}$ acetic acid in the infusion liquid (resulting $\mathrm{pH} 8$ ) as a relevant concentration for evaluation. The experiments were again conducted on all four interfaces. The results are shown in Table 2.

The use of octadecylamine was only partly investigated (data not shown). Aside from the poor signal intensity as compared to dodecylamine (ESI), octadecylamine was also avoided because of contamination of the mass spectrometer, which only disappeared after a long wash period. Therefore, we did not further consider this compound useful for continuous introduction in the MS with the mobile phase. All interfaces were able to generate primary amine adduct ions [PAC + $\operatorname{PrAm}+\mathrm{H}^{+}$. Nevertheless, some differences were perceptible.

Infusion with primary amines on the M-8000. For the M-8000 LC-MS system with SSI interface, the percentage of amine adduct increased with increasing chain length, except for methylamine, the most polar primary amine (Table 2). The most pronounced formation of a primary amine adduct was observed with the most apolar one, i.e., dodecylamine (DDA).

The electrospray results on the same instrument (M-8000) resulted in higher signal intensity compared to SSI. Methylamine this time did not produce a higher percentage of the amine adduct than propylamine. Similar to SSI, dodecylamine gave the highest relative response $(76 \%)$ as primary amine adduct ion (Table 2 ). This condition resulted in the most preferred situation for the M-8000 and was further optimized. Different concentrations were tested (Figure 1). As expected, a gradual decrease in sensitivity was noticed with increasing concentration of the additive. Simultaneously, a small increase in percentage of the amine adduct ion was noticed up to $0.5 \mathrm{mM}$. A good compromise was established at a concentration of $0.1 \mathrm{mM}$. The ratio with between dodecylamine and acetic acid was also considered as a parameter to optimize (Figure 2). A 1:1 ratio seemed comparable to an excess of dodecylamine, but the former was preferred for reasons of stability of the column and compound. An excess of acetic acid yielded decreased sensitivity and relative response of the amine adduct ion. In addition, 15-crown-5 ether, imidazole, 


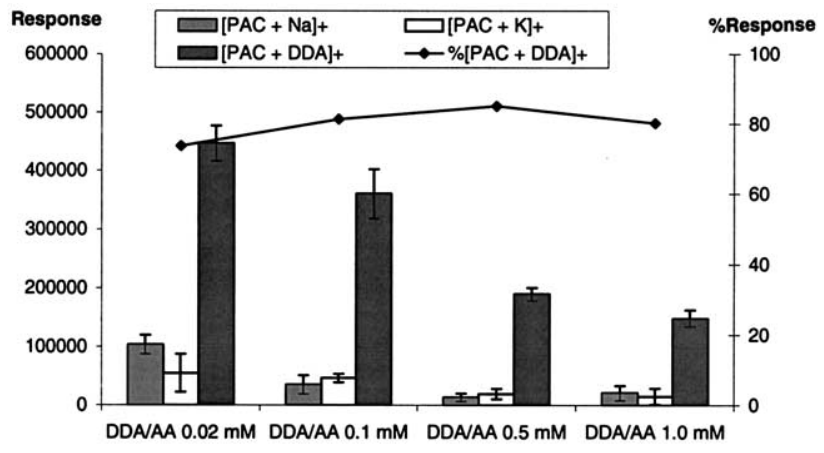

Figure 1. Effect of concentration of DDA/AA on the absolute and relative response and of the paclitaxel adduct ions on the M-8000 after infusion. Error bars indicate $2 \times$ standard deviation $(n=3)$.

piperidine, EDTA and triethylamine were also evaluated by infusion experiments on the M-8000 with ESI, but none resulted in a pronounced signal for paclitaxel or any of its adduct ions. Infusion experiments with small amounts of sodium acetate/acetic acid (0.7/9.3 $\mathrm{mM}$ and $3.6 / 6.4 \mathrm{mM}$ ) to produce the sodium adduct in a reproducible way were carried out, but intense cluster ions $\left[\left(\mathrm{CH}_{3} \mathrm{COONa}\right)_{n}+\mathrm{Na}\right]^{+}$appeared. Furthermore, the potassium adduct was still present in the spectrum.

Infusion with primary amines on the QTOF. On the QTOF system, the ESI interface gave similar results as on the M-8000. It has to be mentioned that all experiments (ESI as well as APCI) on this system made use of cone gas for ionization. This was supported by experiments, which indicated that amine adducts were preferentially formed in this way.

Increasing the carbon tail of the primary amine results in a higher relative response for the [PAC + $\operatorname{PrAm}+\mathrm{H}]^{+}$ion up to nonylamine (Table 2). As electrospray ionization produces ions mainly in the liquid phase, we believe that an explanation therefore should be based on liquid phase processes. We suggest two theories that do not exclude one another. On the one hand, the very hydrophobic paclitaxel molecule could have a high affinity for hydrophobic, longer chain

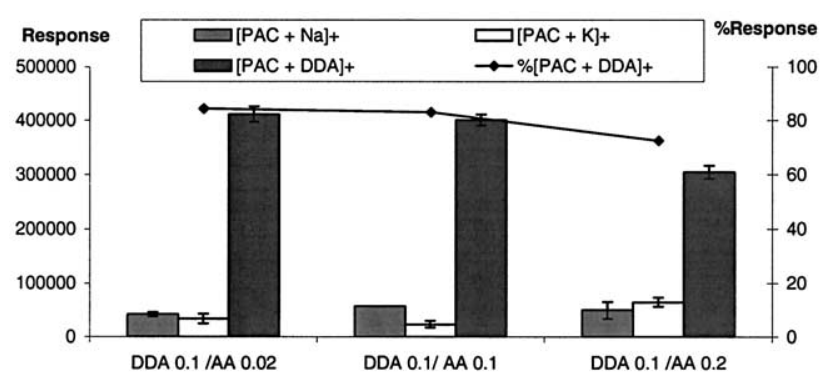

Figure 2. Effect of varying ratio dodecylamine (DDA) and acetic acid (AA) on the absolute and relative response of the paclitaxel adduct ions after infusion (M-8000). Error bars indicate $2 \times$ standard deviation $(n=3)$. Concentrations DDA and AA are given in $\mathrm{mM}$.

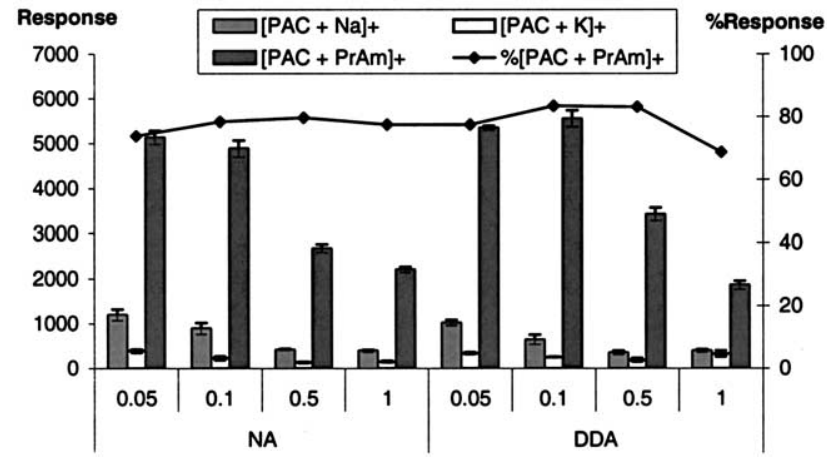

Figure 3. Effect of concentration primary amine/acetic acid $(\mathrm{mM})$ on the absolute and relative response of paclitaxel ion adducts for nonylamine (NA) and dodecylamine (DDA) after infusion (QTOF). Error bars indicate $2 \times$ standard deviation $(n=$ 3).

primary amines in the electrospray droplets, leading to more paclitaxel primary amine complexes amenable to ionization. On the other hand, the complexes with long chain primary amines (hydrophobic tail) could have a higher affinity for the air-liquid interface of the ESI droplets, due to enhanced surface activity. As the fraction of complexes on this droplet surface increases, the response should simultaneously increase. The latter has been suggested by Cech and Enke, who described that tripeptides with a more hydrophobic side-chain gave better ionization, probably due to increased surface activity [15].

Based on the results in Table 2, dodecylamine at a concentration of $1.0 \mathrm{mM}$ gives a lower absolute and relative response as $[\mathrm{PAC}+\operatorname{PrAm}+\mathrm{H}]^{+}$than nonylamine and hexylamine at first sight. At a concentration of $0.1 \mathrm{mM}$ primary amine, however (Figure 3), dodecylamine results in the same or even better sensitivity than nonylamine, but gives a slightly higher relative response ( 83.5 vs. $78.4 \%)$. We believe that the decreasing absolute response of $[\mathrm{PAC}+\operatorname{PrAm}+\mathrm{H}]^{+}$at increasing concentrations of primary amine is caused by steric hindrance at the droplet surface. Assuming this, and knowing that steric hindrance is more likely to occur with the larger molecule dodecylamine, it seems evident that lowering the concentration of dodecylamine reduces steric hindrance and results in similar or even superior ionization efficiency as compared to nonylamine. Therefore, dodecylamine at a concentration of $0.1 \mathrm{mM}$ (with acetic acid $0.1 \mathrm{mM}$ ) was considered as the preferred primary amine for addition to the mobile phase.

Finally, the APCI source on the QTOF was evaluated for its suitability to generate primary amine adduct ions (Table 2). The ionization efficiency of APCI was inferior to ESI. In addition, the relative response of the adduct ion never exceeded $30 \%$, and only a minor trend towards an increased response of $[\mathrm{PAC}+\operatorname{PrAm}+\mathrm{H}]^{+}$ with increasing chain length of the primary amine was observed. This supports the statement that adduct formation of paclitaxel with primary amines is mainly a 


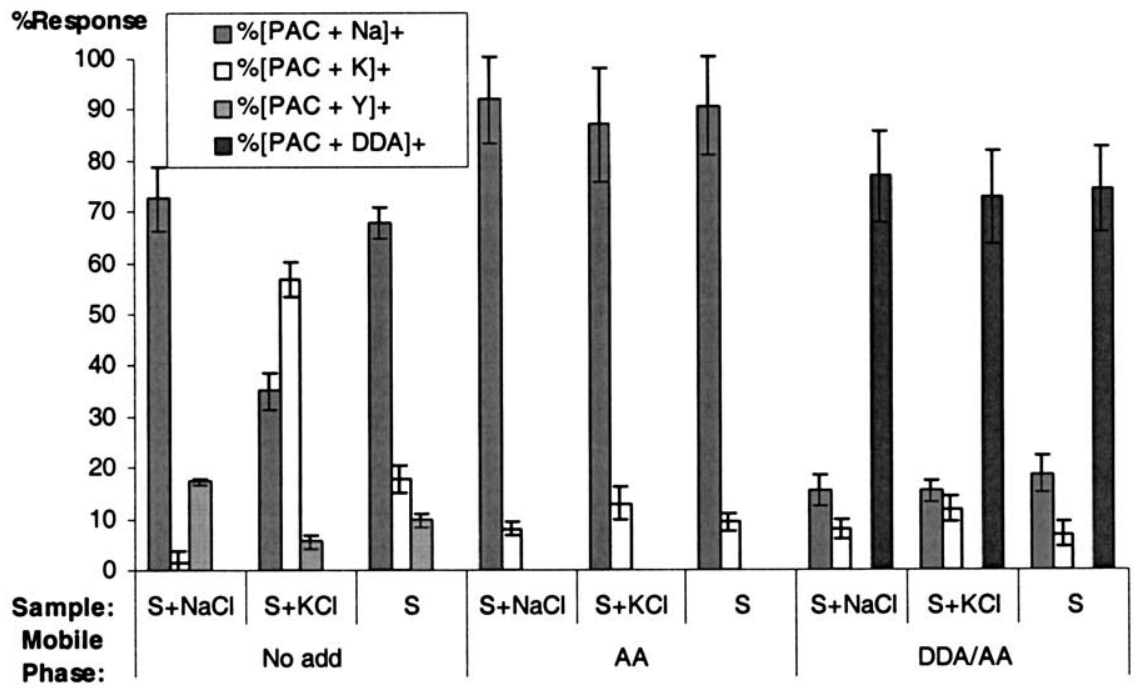

Figure 4. Effect of mobile phase and alkali metal in the sample on the absolute response and reproducibility of paclitaxel adduct ions after LC-MS (ESI - M-8000). Error bars indicate $2 \times$ standard deviation $(n=8)$.

liquid phase process. As APCI is more a gas phase process, enhanced "surface activity" on the ESI droplet does not necessarily increase the response with this type of ionization. Based on these results, we consider the combination of APCI and primary amines less suitable for our application. We concluded from these experiments that ESI is preferred to APCI for the creation of primary amine adduct ions and focused our attention to this interface for further experiments.

\section{Chromatographic evaluation with LC-ESI-MS}

The results obtained by infusion were evaluated by LC-ESI-MS on both the M-8000 and QTOF. This approach will verify whether the results obtained by the infusion can be extrapolated to a more real-life situation, i.e., the LC-ESI-MS analysis of samples of varying composition. The following experiment was therefore designed. Paclitaxel was diluted to $1000 \mathrm{ng} / \mathrm{mL}$ from stock solutions in mobile phase and this solution was divided into three portions. $\mathrm{NaCl}(17 \mathrm{mM})$ was added to the first sample $(\mathrm{S}+\mathrm{NaCl}), \mathrm{KCl}(4 \mathrm{mM})$ to the second $(\mathrm{S}+\mathrm{KCl})$, and nothing to the third sample (S), this to mimic real samples containing varying amounts sodium and potassium chloride. The samples were eight times cross injected ( $5 \mu \mathrm{L}$ or $5 \mathrm{ng}$ on-column) onto the column. In this way, the retained paclitaxel (capacity factor $\mathrm{k}^{\prime}=3.6$ ) was separated from unretained substances like minerals and the added alkali metals. This experiment was repeated with different mobile phases. The main consideration was a reproducible formation of one ion, despite this variable salt concentration in the sample.

Chromatographic evaluation with LC-ESI-MS on M-8000. The results of these LC-ESI-MS experiments on the M-8000 are represented in Figure 4. Three different mobile phases were compared: (1) $\mathrm{MeOH} /$ water $(70 / 30$ by vol.) without additive (no add), (2) acetic acid (1.7 mM) as additive (AA), and (3) acetic acid/dodecylamine (0.1 $\mathrm{mM} / 0.1 \mathrm{mM}$ ) as additive (DDA/AA). Aside from the sodium and potassium adduct, another unknown adduct at $\mathrm{m} / \mathrm{z} 974$ was noted and further named [PAC + $\mathrm{Y}]^{+}$. Although MS/MS experiments indicate that this ion consists of paclitaxel with at least two sodium ions attached (fragmentation of $\mathrm{m} / \mathrm{z} 974$ to 898 , i.e., [PAC + $2 \mathrm{Na}-\mathrm{H}]^{+}$) we could not determine the exact origin of it. Without additive in the mobile phase, a large variability in response between samples is noted. Analysis of the alkali metal free sample (S) and the sample spiked with $\mathrm{NaCl}(\mathrm{S}+\mathrm{NaCl})$ results in about $72 \%$ of all paclitaxel ions present in the form of $[\mathrm{PAC}+\mathrm{Na}]^{+}$, while for the sample containing potassium $(\mathrm{S}+\mathrm{KCl})$, only $32 \%$ of the total paclitaxel ions consisted of $[\mathrm{PAC}+\mathrm{Na}]^{+}$. The amount of potassium in the sample had a great influence and resulted in $58 \%[\mathrm{PAC}+\mathrm{K}]^{+}$of the total response. Despite the chromatographic separation, the presence of different types of alkali metals in the sample gave different patterns of adduct formation. Such conditions are not suitable for quantitative analysis.

With acetic acid alone in the mobile phase $(1.7 \mathrm{mM})$, a pronounced formation of $[\mathrm{PAC}+\mathrm{Na}]^{+}$was observed. Absolute and relative response of $[\mathrm{PAC}+\mathrm{Na}]^{+}$are of the same order of magnitude. The results of this chromatographic evaluation are somewhat conflicting with the infusion experiments for acetic acid. More potassium adduct was noted with the infusion method. Perhaps, the potassium was originating from the standard solution or the syringe, and is less present in the mobile phase used for chromatography. This points out that infusion might not be the only way to evaluate 


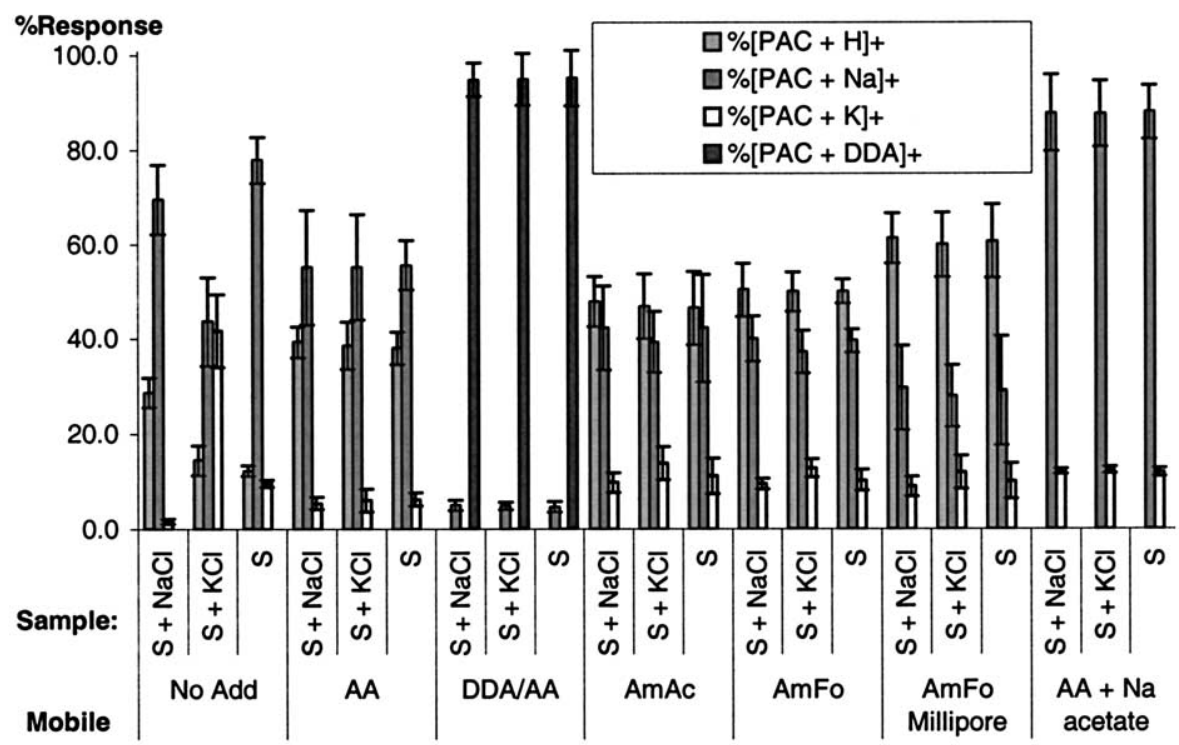

Figure 5. Effect of mobile phase and alkali metal in the sample on the relative response of paclitaxel adduct ions after LC-MS (ESI - QTOF, $n=8$ ).

adduct formation and chromatography can definitely modify the adduct formation.

A mobile phase containing dodecylamine/acetic acid $(0.1 \mathrm{mM} / 0.1 \mathrm{mM})$ also provides reproducible results. When comparing the different samples, both absolute and relative responses of the base peak [PAC $+\mathrm{DDA}]^{+}$were closely related, with confidence intervals overlapping and of the same order of magnitude. Eventually, we consider both $\mathrm{MeOH} /$ water $(70 / 30$, by vol) with acetic acid $(1.7 \mathrm{mM})$ or with dodecylamine/ acetic acid $(0.1 \mathrm{mM} / 0.1 \mathrm{mM})$ acceptable mobile phases for quantitation on the M-8000 with the ESI interface.

Chromatographic evaluation with LC-ESI-MS on QTOF. A more comprehensive evaluation of mobile phases by LC-ESI-MS was performed on the QTOF (Figure 5). The same strategy was followed, so again different mobile phases were used to analyze the three samples ( + $\mathrm{NaCl}, \mathrm{S}+\mathrm{KCl}, \mathrm{S}$ ) with the same paclitaxel concentration but with varying alkali metal content. Without additive in the mobile phase $(\mathrm{MeOH} /$ water, $70 / 30$ by vol.), the results were again not suitable for quantitation. The adduct ion with the most pronounced response was again $\left[\mathrm{PAC}+\mathrm{Na}^{+}\right.$, but large variations in relative response between samples were observed.

When adding only acetic acid to the mobile phase $(1.7 \mathrm{mM})$, the signal was divided over two main adduct ions. Between 38 and $39.3 \%$ of all paclitaxel ions originated from $[\mathrm{PAC}+\mathrm{H}]^{+}$and about $55 \%$ was present in the form of $[\mathrm{PAC}+\mathrm{Na}]^{+}$, and this in a reproducible way, within and between the different samples. Only a small amount $(6 \%)$, was present in the form of [PAC + $\mathrm{K}]^{+}$. Comparing these results obtained by LC-ESI-MS to the results obtained by syringe infusion again revealed some differences in adduct formation. Infusion from a syringe resulted in much more potassium adduct ion
$(24 \%)$, so the chromatographic separation diminished the potassium adduct ion to about $6 \%$. Again, these chromatographic conditions decreased the effect of potassium but not of sodium on the ionization. Nevertheless, a situation where the response is divided into two signals is not favorable for sensitive MS/MS analysis.

Adding dodecylamine/acetic acid to the mobile phase got the reproducibility to a level acceptable for quantitation, with more than $94.9 \%$ of all paclitaxel ions present in the form of $[\mathrm{PAC}+\mathrm{DDA}]^{+}$in all three samples.

An attempt was made to enhance the signal of [PAC $+\mathrm{H}]^{+}$by switching to ammonium acetate $(1.6 \mathrm{mM} ; \mathrm{pH}$ $4.75)$ or ammonium formate $(1.6 \mathrm{mM}$; $\mathrm{pH} 3.75)$ as additive. This enhanced the relative signal intensity of $[\mathrm{PAC}+\mathrm{H}]^{+}$only slightly (ammonium acetate $47 \%$, ammonium formate: $50 \%$ ). Another effort to increase the signal intensity of $[\mathrm{PAC}+\mathrm{H}]^{+}$was the use of ultra distilled water (with Millipore Synergy 185) and rinsing of the glassware containing the mobile phase with concentrated formic acid. With ammonium formate as additive, the combination of these alterations enhanced the relative response of $[\mathrm{PAC}+\mathrm{H}]^{+}$to $61 \%$. Although reproducible, a more pronounced relative response would be desirable, but was not achieved. A more pronounced ionization of another ion, namely [PAC + $\mathrm{Na}]^{+}$, was strived for by the addition of $20 \mu \mathrm{M}$ sodium acetate to $1.7 \mathrm{mM}$ acetic acid. Relative responses of around $88 \%$ were reached for all samples with excellent reproducibilities. This low amount of the non-volatile sodium acetate did not produce any source contamination.

In conclusion, for the ESI-QTOF, different mobile phases enabled to obtain reproducible results. The use of additives in the mobile phase again appeared to be a necessity. All additives improved the reproducibility 
between samples considerably, but some also resulted in a pronounced formation of one single ion. The highest relative response noticed for $[\mathrm{PAC}+\mathrm{H}]^{+}$amounted to $61 \%$. A pronounced relative response occurred with of dodecylamine/acetic acid as $[\mathrm{PAC}+\mathrm{DDA}]^{+}$and with acetic acid/sodium acetate as $[\mathrm{PAC}+\mathrm{Na}]^{+}$.

\section{Conclusion}

Adduct formation of paclitaxel was studied under various conditions. Infusion with a syringe and LC-MS analysis were both used for the evaluation of adduct formation. After comparison of these techniques, it became clear that LC-MS gives closer representation of the real situation. Although fast and easy and therefore still useful for an initial screening, infusion could not eliminate the influence of residual alkali metals coming from syringe or sample. Higher quantities of [PAC + $\mathrm{K}]^{+}$were often observed with syringe infusion. LC on the other hand was more influenced by residual sodium in the system and as a result gave higher signal for $[\mathrm{PAC}+\mathrm{Na}]^{+}$. Two different MS systems (M-8000 and QTOF) were compared which demonstrated similar trends but at the same time notable differences. Electrospray seemed to be the most suitable source for our application on both instruments. With ammonium formate as additive on the QTOF, electrospray sometimes resulted in $[\mathrm{PAC}+\mathrm{H}]^{+}$while this was never the case on the M-8000. With primary amines as additive, increasing chain length resulted in higher relative responses $[\mathrm{PAC}+\operatorname{PrAm}+\mathrm{H}]^{+}$on both instruments. Experiments using chromatography clearly showed that additives are a necessity; without additive in the mobile phase, paclitaxel adduct formation was strongly influenced by the alkali metal content of the sample, despite the chromatographic process. Addition of the selected primary amine, dodecylamine, to the mobile phase proved to be successful to convert almost all paclitaxel adduct ions to $[\mathrm{PAC}+\mathrm{DDA}]^{+}$on both instruments. The more common additives like acetic acid, ammonium formate and ammonium acetate resulted in a partition of the signal between $\left[\mathrm{PAC}+\mathrm{H}^{+}\right.$ and $[\mathrm{PAC}+\mathrm{Na}]^{+}$on the QTOF. Reproducible and pronounced formation of one ion was noticed with dodecylamine/acetic acid and with acetic acid/sodium acetate on this instrument. These results show that different mobile phase additives can be used and can result in different ions. Thus, several suitable possibilities exist to develop a quantitative method. Furthermore, the presented work also illustrates the importance of evaluating any variability in adduct formation during method development.

\section{Acknowledgments}

This work was supported by grant GOA99-120501.99 and a grant from the Centrum voor Studie en Behandeling van Gezwelziekten, Ghent. The authors thank Ms. Sofie Vande Casteele for technical assistance and gratefully acknowledge assistance from Merck KGaA Darmstadt and support from VWR Belgium.

\section{References}

1. Matuszewski, B. K.; Constanzer, M. L.; Chavez-Eng, C. M. Matrix Effect in Quantitative LC/MS/MS Analyses of Biological Fluids: A Method for Determination of Finasteride in Human Plasma at Picogram per Milliliter Concentrations. Anal. Chem. 1998, 70, 882-889.

2. Mei, H.; Hsieh, Y. S.; Nardo, C.; Xu, X. Y.; Wang, S. Y.; Ng, K.; Korfmacher, W. A. Investigation of Matrix Effects in Bioanalytical High-Performance Liquid Chromatography/Tandem Mass Spectrometric Assays: Application to Drug Discovery. Rapid Commun. Mass Spectrom. 2003, 17, 97-103.

3. Bonfiglio, R.; King, R. C.; Olah, T. V.; Merkle, K. The Effects of Sample Preparation Methods on the Variability of the Electrospray Ionization Response for Model Drug Compounds. Rapid Commun. Mass Spectrom. 1999, 13, 1175-1185.

4. Choi, B. K.; Gusev, A. I.; Hercules, D. M. Postcolumn Introduction of an Internal Standard for Quantitative LC-MS Analysis. Anal. Chem. 1999, 71, 4107-4110.

5. Li, X. F.; Ma, M. S.; Scherban, K.; Tam, Y. K. Liquid Chromatography-Electrospray Mass Spectrometric Studies of Ginkgolides and Bilobalide Using Simultaneous Monitoring of Proton, Ammonium, and Sodium Adducts. Analyst 2002, 127, 641-646.

6. Ortelli, D.; Rudaz, S.; Cognard, E.; Veuthey, J. L. Analysis of Dihydroartemisinin in Plasma by Liquid Chromatography Mass Spectrometry. Chromatographia 2000, 52, 445-450.

7. Teshima, K.; Kondo, T.; Maeda, C.; Oda, T.; Hagimoto, T.; Tsukuda, R.; Yoshimura, Y. Application of 1-Alkylamines to a Liquid Chromatographic/Turbo Ionspray Tandem Mass Spectrometric Method for Quantifying Metabolites of a New Bone Anabolic Agent, TAK-778, in Human Serum. J. Mass Spectrom. 2002, 37, 631-638.

8. Stefansson, M.; Sjoberg, P. J. R.; Markides, K. E. Regulation of Multimer Formation in Electrospray Mass Spectrometry. Anal. Chem. 1996, 68, 1792-1797.

9. Lopes, N. P.; Stark, C. B. W.; Hong, H.; Gates, P. J.; Staunton, J. Z. A Study of the Effect of $\mathrm{pH}$, Solvent System, Cone Potential, and the Addition of Crown Ethers on the Formation of the Monensin Protonated Parent Ion in Electrospray Mass Spectrometry. Analyst 2001, 126, 1630-1632.

10. Greig, M.; Griffey, R. H. Utility of Organic-Bases for Improved Electrospray Mass-Spectrometry of Oligonucleotides. Rapid Commun. Mass Spectrom. 1995, 9, 97-102.

11. Jemal, M.; Almond, R. B.; Teitz, D. S. Quantitative Bioanalysis Utilizing High-Performance Liquid Chromatography Electrospray Mass Spectrometry via Selected Ion Monitoring of the Sodium Ion Aadduct $[\mathrm{M}+\mathrm{Na}](+)$. Rapid Commun. Mass Spectrom. 1997, 11, 1083-1088.

12. Alexander, M. S.; Kiser, M. M.; Culley, T.; Kern, J. R.; Dolan, J. W.; McChesney, J. D.; Zygmunt, J.; Bannister, S. J. Measurement of Paclitaxel in Biological Matrices: High-Throughput Liquid Chromatographic-Tandem Mass Spectrometric Quantification of Paclitaxel and Metabolites in Human and Dog Plasma. J. Chromatogr. B Anal. Technol. Biomed. Life Sci. 2003, 785, 253-261.

13. Thurman, E. M.; Ferrer, I.; Barcelo, D. Choosing Between Atmospheric Pressure Chemical Ionization and Electrospray Ionization Interfaces for the HPLC/MS Analysis of Pesticides. Anal. Chem. 2001, 73, 5441-5449.

14. Zhou, S. L.; Hamburger, M. Effects of Solvent Composition on Molecular Ion Response in Electrospray Mass Spectrometry: Investigation of the Ionization Processes. Rapid Commun. Mass Spectrom. 1995, 9, 1516-1521.

15. Cech, N. B.; Enke, C. G. Relating Electrospray Ionization Response to Nonpolar Character of Small Peptides. Anal. Chem. 2000, 72, 2717-2723. 